\title{
Dental Amalgam - A Controversial Filling Material
}

\section{Syed Kashif Abrar SKA ${ }^{1 *}$, Raheel Allana RA², Syed M Abdullah Salman SMAS ${ }^{3}$, Hassaan-ul-Qamar HQ ${ }^{3}$, Ather $\mathrm{Akber}^{2}$ $\mathrm{AA}^{4}$ and Lovekesh Kumar $\mathbf{L K}^{5}$}

${ }^{1}$ Department of Periodontology, Dow Dental College, Dow University of Health Sciences, Karachi Pakistan ${ }^{2}$ Department of Epidemiology and Biostatistics, School of Public Health, Dow University of Health Sciences, Karachi Pakistan ${ }^{3}$ Department of Oral Medicine, Dow Dental College, Dow University of Health Sciences, Karachi Pakistan ${ }^{4}$ Department of Periodontology, Dow International Dental College, Dow University of Health Sciences, Karachi Pakistan ${ }^{5}$ Department of Oral Medicine and Diagnosis, Dow Dental College, Dow University of Health Sciences, Karachi Pakistan *Corresponding Author: Syed Kashif Abrar SKA, Department of Periodontology, Dow Dental College, Dow University of Health Sciences, Karachi Pakistan.

Received: November 22, 2019; Published: November 29, 2019

DOI: $10.31080 /$ ASDS.2019.03.0718

\section{Abstract}

Dental Amalgam is being used in the field of Dental Sciences for more than 150 years. It is the most versatile and excellent filling material and it is still being used due to its low cost, its durability, its resistance to further decay and its strength. It is produced by mixing of mercury with particles such as copper, tin, silver, indium, palladium etc. to produce a workable mass of Amalgam Alloy. The most important controversy which lies with Amalgam and is still being tagged with dental amalgam is the release of mercury vapors from the Amalgam fillings. Exposure to mercury can occur from multiple amount of sources which includes Atmosphere, drinking water, diet including fish etc. Dental Amalgam fillings releases mercury during mixing, setting, polishing and removal of the desired filling but once the material is set very minute quantities of mercury vapors are released which is far below the current health standard. Researchers do believe that Amalgam produces mercury but no such significant changes have been found till date. At Present there is no scientific evidence regarding the discharge of mercury from dental amalgam fillings that can cause or worsen neurological diseases such as Alzheimer's disease and Parkinson's disease etc. Though there are evidences that Amalgam causes some hypersensitivity reactions in the oral mucosa. The FDA have investigated in the past regarding its safety and that have placed no restrictions on its use.

Keywords: Mercury; Amalgam; Toxicity; Safety; Controversy

\section{Introduction}

Dental Amalgam is a metal alloy mixture along with mercury. Mercury is mixed with solid alloy particles which produces a workable mass. The reaction between the mercury and the alloy particles is known as Amalgamation. Dental Amalgam restorations are easy to insert, they have resistance to fracture, prevents marginal leakage and have a relatively long life. The draw backs of dental amalgam includes their silver-grey appearance, corrosion and galvanic action, brittle nature, less desirable, because metals can conduct hot and cold temperature there may be a little sensitivity to hot and cold [1]. Despite its many advantages and disadvantages, concerns regarding its toxicity is still an unsolved question.

\section{Historical background of dental amalgam}

It's being used since many years with wide variety of success and it is one of the conventionally used filling material [5]. Evidence regarding its use begin in the Tang Dynasty era than in 1833 the
Crawcour brothers introduced this material to United States. In 1890 G.V Black proposed the formula for Dental Amalgam. Amalgam consists of alloys of tin, silver, zinc which are combined with mercury forming two phases named as gamma. The gamma-2 phase is the weakest phase which is responsible for the early degeneration of the filling material. Copper was than introduced which replaces this weakest phase and is replaced by copper tin phase which is comparatively stronger than the mercury tin phase [14].

\section{Sources of mercury exposure}

Mercury exists in nature in the form of elemental mercury and it is mostly found in the earth's crust [2]. Exposure of mercury can occur from many sources. Mercury can be exposed through air, food which are contaminated. Only small amounts of mercury will enter the stomach if it's ingested. Vapors of mercury when inhaled, about $80 \%$ will enter blood and then it goes to the other parts of 
the body. In the body mercury stays there for weeks or months. Mercury can also be transferred from pregnant women to the child who is being developed [7].

\section{Exposure of mercury from amalgam fillings}

Mercury exposure can occur through amalgam fillings [3]. Mercury vapors may be released into the atmosphere and oral cavity during the process of mixing (trituration), condensation and removal of the old grey colored fillings [5,6]. Chewing and taking hot beverages can also cause out glassing of mercury from the filling [4]. Mercury quantity releasing from the amalgam fillings ranges from 2 to 28 micrograms/facet/surface/day [2]. The World Health Organization has stated that sea food like fish can increase mercury urine level to 5 - $20 \mu \mathrm{g} / \mathrm{L}$ which is more than the amount of mercury released from amalgam [1]. When the reaction of amalgam is completed very less amount of mercury is released [3] and the amount of out glassing of mercury can be reduced by good condensation of the material [10]. M Azarsina., et al. conducted a study based on the hypothesis that polishing an amalgam surface will release less mercury. Amalgam fillings were polished with carbamide peroxide gel and it was found that less mercury is released from the polished surface [15].

\section{Forms of mercury}

Environmental mercury occurs naturally and it has many forms including organic and inorganic compounds [1].

\section{Elemental mercury}

It is pure and is in its uncombined form. It is liquid at room temperature. If it is not sealed of it evaporates and forms a vapor E.g. in thermometers and electrical switches.

\section{Organic mercury}

Commonly found in fish and the most toxic are the methyl and ethyl mercury compounds $[1,2]$. It is formed when mercury combines with the carbon and the other elements. The next in line is the mercury vapor [1].

\section{Inorganic mercury}

They are the least toxic form of mercury [1]. Most of them are white powder or crystals.

\section{Health effects of mercury in children}

Many trials has been done in children to assess the mercury vapors toxicity.

A randomized clinical trial was done in 507 children in Lisbon to assess the safety of this filling material. Half of them received amalgam as a posterior filling and half of them received composite. Neuro-behavioral assessments were done and the authors found that statistically there was no difference in neuro behavioral assessment and nerve conduction velocities when amalgam receiving children were compared with those children who had composite fillings. Hence the authors came to conclusion that amalgam is safer and still is reliable to be use as a filling material [8].

Lars Bar regard assessed the effect of amalgam on renal tubular function at a molecular level. Children 6 - 10 years with caries on posterior teeth were included in this experimental trial. This clinical trial did not find any negative correlation between amalgam and renal tubular function. However Increase in micro albuminuria was a clinical finding but it may be a chance finding so it should be further tested [9].

Xibiao Ye conducted a trial in children aged between 7 - 11 years to find out the effects of dental amalgam on neuro-behavioral and neuro-psychological performance of children. Laboratory analysis were done for urine samples, and two renal biomarkers namely Albumin and N-acetyl -B- D- glycosaminidase were measured. For Behavioral analysis, Child Behavior Checklist scale was used and comparison was made between children having amalgam filling and amalgam free children. Statistically there were no difference between the two groups of children. Although urine mercury level was high in children with amalgam fillings, but it had no effect on neuro-behavioral and neuro-psychological effect on children [10].

\section{Health effects of mercury in adults}

Halbach assessed the dose of absorbed mercury from dental amalgam in a randomized control trial. Results from this trial showed that the absorbed dose of mercury from dental amalgam was still low as compared to the tolerable dose of Mercury which is $30 \mu \mathrm{g}$ which is given by WHO in 1999 [11].

Bailer J assessed the toxicological and the psychological effects of mercury exposure through dental fillings. For this purpose, a screening questionnaire was used. And the amalgam sensitive subjects (those subjects who believed that these silver grey fillings have made them ill) were compared with amalgam non-sensitive subjects. The study did not provide any evidence of symptoms of patients due to mercury and these symptoms were totally due to the psychological factors [12].

\section{Hypersensitivity reactions from dental amalgam}

Studies which have been done regarding the toxicity of amalgam does not clearly show about its adverse effect on human body but amalgam can cause considerable amount of hypersensitivity reactions in the oral mucosa. Most common reaction is Coombs type 4 hypersensitivity or contact dermatitis which has reactions less than $1 \%$ [14] other reaction is the lichenoid reaction. Lichenoid reaction is a cell mediated response and is usually caused by acquisition of salts of mercury in the oral mucosa causing a type 
4 hypersensitivity reaction. This reaction causes reticular white plaques or ulceration, this ulceration or plaques require no treatment and will resolve on its own or on removal of the desired amalgam filling [10].

\section{Occupational exposure in dental professionals}

Mercury exposure through amalgam fillings is very common in dental professionals. Given the fact that Mercury is toxic and has got some drastic potential effects on human body, dental practices should adhere to the proper mercury handling in their practices.

Common sources of contamination from mercury occurs from amalgam scrap, spilling of mercury etc. Mercury spills are thought to be one of the dangerous amongst them which can cause serious effects. There are many studies and researches done regarding the exposure of mercury in dental professionals. Chaari N., et al. conducted a study regarding the penetration of mercury spilling in dental professionals. This study was conducted in Tunisia. Chari $\mathrm{N}$ and his team conducted a cross sectional study on 52 dentists and dental assistants working in private hospital setup. A control group of same sample size was taken. This study was questionnaire based which included certain parameters such as handling of mercury and its techniques, work environment, prevention of hygiene measures, non-professional exposure of mercury. Based on this questionnaire they found out that $60 \%$ of the professionals that are working in a dental setup have an increased risk of exposure. Their urinary and salivary mercury levels were increased as compared to the control group. Certain factors were present which led to the higher exposure of mercury in case group. This included the mercury storage in open containers, Amalgam waste not properly disposed and was inadequate and their hygiene practices which include eating lunch at the work place, this all led to the higher percentage of mercury exposure in case group [13].

\section{Management of mercury waste in dental setup}

There are many certain and important measures that should be adopted by every dental personal in order to get avoid from excess mercury exposure.

- Work in well ventilated areas.

- Mercury should be stored in closed and tightly sealed containers.

- Capsules for amalgamation should be tightly closed.

- Avoid heating mercury.

- Avoid carpeting the dental setup as its decontamination is quite difficult.

- Clean the spilled mercury quickly. Droplets should be picked up by a narrow bore tubing (via a washer bottle trap).

- Use water spray and suction while grinding amalgam fillings.

- Periodically check mercury vapor levels in dental operatory.
- Store and salvage the amalgam scrap in water containing sodium thiosulphate.

- Alert all dental personal who handle mercury regarding its potential hazards and necessity for observing and practicing good hygiene practice [1].

Amalgam substitutes

Though Amalgam is still a controversial subject, many recent fillings have played their part as an alternative to amalgam. One such material is Composite resin fillings. They are tooth colored and white fillings which are reinforced by a filler with a coupling agent. They serve better than amalgam when small occlusion filling is required. A compare to amalgam, composite is less durable and technique sensitive and has a higher cost. Another material is a glass ionomer filling material. Though the use of amalgam has been limited in certain areas due to its potential hazards, more studies and researches are required on safety of the alternatives before they can be used as a substitute [3].

\section{Conclusion}

From the recent studies and researches it is evident that amalgam has not posed any serious or catastrophic health hazard and does not cause any systemic toxicological effect [4] except it has some allergic reaction. From this evidence we can conclude that amalgam is safe and can be used if mercury hygiene recommendations must be properly followed in order to get minimal exposure from mercury.

\section{Authors' Contributions}

All the Authors i.e. S.K.A, R.A, S.M.A.S, H.Q, A.A and L.K contributed to the manuscript of this review article.

\section{Bibliography}

1. Robert G Craig. "Restorative Dental Materials". Tenth Edition, Mosby-Year Book Inc., (1960): 209-234.

2. Robin A. "Benhoft, Mercury Toxicity and Treatment: A Review of the Literature”. J. Environ. Public Health (2012).

3. Rathore M., et al. "The Dental Amalgam Toxicity Fear: A Myth or Actuality". Toxicology International. 2012; 19.2 (2012): 8188.

4. Uçar Y and Brantley WA. "Biocompatibility of Dental Amalgams". International Journal of Dentistry (2011): 981595.

5. JF Mc Cabe and AWG Walls. Applied Dental Materials, Eighth Edition, (2006): 157.

6. $\quad$ McParland H and Warnakulasuriya S. "Oral Lichenoid Contact Lesions to Mercury and Dental Amalgam-A Review”. Journal of Biomedicine and Biotechnology (2012): 589-569. 
7. Chunhan Ngim and Allister Daquan Ngim. "Health and safety in the dental clinic- Hygiene regulations for the use of elemental mercury in the protection of rights, safety and well-being of the patients, workers and the environment". Singaore Dental Journal (2013).

8. DeRouen TA., et al. "Neurobehavioral effects of dental amalgam in children: a randomized clinical trial". JAMA. 295.15 (2006): 1784-1792.

9. Barregard L., et al. "Renal effects of dental amalgam in children: the New England children's amalgam trial". Environ Health Perspect 116 (2008): 394-399.

10. Ye X., et al. "Nephrotoxicity, Neurotoxicity, and Mercury Exposure among Children with and without Dental Amalgam Fillings". International Journal of Hygiene and Environmental Health. 212.4 (2009).

11. Halbach S., et al. "Blood and urine mercury levels in adult amalgam patients of a randomized controlled trial: interaction of Hg species in erythrocytes". Environ Res. 107 (2008): 69-78.

12. Bailer J., et al. "Adverse health effects related to mercury exposure from dental amalgam fillings: toxicological or psychological causes?" Psychol Med. 31 (2001): 255-263.

13. Chaari N., et al. "Mercury impregnation in dentists and dental assistants in Monastir city, Tunisia”. Rev Stomatol Chir Maxillofac 110 (2009):139-44.

14. Kenneth J Anusovice., et al. ". "Philips Science of Dental Materials". 12th ed. Elsevier Sciences (2012): 359.

15. Azarsina M., et al. "Effect of Surface Polishing on Mercury Release from Dental Amalgam After Treatment 16\% Carbamide Peroxide Gel". Journal of Dentistry (Tehran, Iran). 8.1 (2011): 33-38.

Volume 3 Issue 12 December 2019

(C) All rights are reserved by Syed Kashif Abrar SKA.,

et al. 